\title{
Measurement of the deuteron polarization in a large target
}

\author{
Spin Muon Collaboration (SMC)
}

B. Adeva ${ }^{a}$, S. Ahmad ${ }^{b}$, A. Arvidson ${ }^{\text {c }}$, B. Badelek ${ }^{\text {c,d }}$, M.K. Ballintijn ${ }^{\mathrm{e}}$, G. Bardin ${ }^{\mathrm{f}}$, G. Baum ${ }^{\mathrm{g}}$, P. Berglund ${ }^{\text {h }}$, L. Betev ${ }^{\text {i }}$, I.G. Bird ${ }^{\text {f, }, 1}$, R. Birsa ${ }^{\text {j }}$, P. Björkholm ${ }^{c}$, B.E. Bonner ${ }^{b}$, N. de Botton ${ }^{f}$, M. Boutemeur ${ }^{k, 2}$, F. Bradamante ${ }^{j}$, A. Bressan ${ }^{j}$, A. Brüll ${ }^{1,3}$, J. Buchanan ${ }^{b}$, S. Bültmann ${ }^{g}$,

E. Burtin ${ }^{\mathrm{f}}$, C. Cavata ${ }^{\mathrm{f}}$, J.P. Chen ${ }^{\mathrm{m}}$, J. Clement ${ }^{\mathrm{b}}$, M. Clocchiatti ${ }^{\mathrm{j}}$, M.D. Corcoran ${ }^{\mathrm{b}}$,

D. Crabb ${ }^{\mathrm{m}}$, J. Cranshaw ${ }^{\mathrm{b}}$, T. Çuhadar ${ }^{\mathrm{n}}$, S. Dalla Torre ${ }^{\mathrm{j}}$, R. van Dantzig ${ }^{\mathrm{e}}$, D. Day ${ }^{\mathrm{m}}$,

S. Dhawan ${ }^{k}$, C. Dulya ${ }^{\circ}$, A. Dyring ${ }^{c}$, S. Eichblatt ${ }^{b}$, J.C. Faivre ${ }^{f}$, D. Fasching ${ }^{p}$, F. Feinstein ${ }^{f}$,

C. Fernandez ${ }^{\mathrm{a}, \mathrm{q}}$, B. Frois ${ }^{\mathrm{f}}$, C. Garabatos ${ }^{\mathrm{a}}$, J.A. Garzon ${ }^{\mathrm{a}, \mathrm{q}}$, T. Gaussiran ${ }^{\mathrm{b}}$, M. Giorgi ${ }^{\mathrm{j}}$,

E. von Goeler ' , I.A. Golutvin ${ }^{\text {j }}$, A. Gomez ${ }^{\mathrm{a}, \mathrm{q}}$, G. Gracia ${ }^{\mathrm{a}}$, M. Grosse Perdekamp ${ }^{\mathrm{o}}$, W. Grübler ${ }^{\text {, }}$

D. von Harrach ", T. Hasegawa ${ }^{v, 4}$, P. Hautle t,5, N. Hayashi v, C.A. Heusch ${ }^{\text {w }}$, N. Horikawa v,

V.W. Hughes ${ }^{k, *}$, G. Igo ${ }^{\circ}$, S. Ishimoto ${ }^{v, 6}$, T. Iwata ${ }^{v}$, M. de Jong ${ }^{x}$, E.M. Kabuss ${ }^{\text {, }}$ R. Kaiser ${ }^{\text {, }}$,

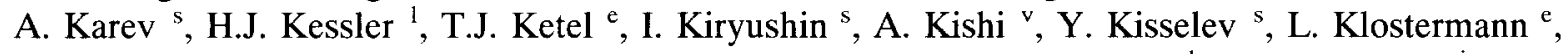

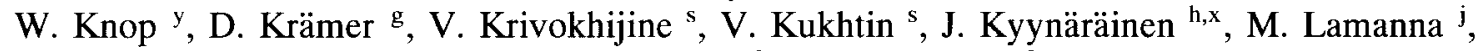

U. Landgraf ', K. Lau ${ }^{\text {q, T. Layda }}{ }^{\text {w }}$, F. Lehar ${ }^{\mathrm{f}}$, A. de Lesquen ${ }^{\mathrm{f}}$, J. Lichtenstadt ${ }^{\mathrm{z}}$, T. Lindqvist ${ }^{\text {c }}$,

M. Litmaath ${ }^{\text {e }}$, S. Lopez-Ponte ${ }^{\mathrm{a}, \mathrm{q}}$, M. Lowe ${ }^{\mathrm{b}}$, A. Magnon ${ }^{\mathrm{f}}$, G.K. Mallot ${ }^{\mathrm{u}, \mathrm{x}}$, F. Marie ${ }^{\mathrm{f}}$, A. Martin ${ }^{\mathrm{j}}$, J. Martino ${ }^{\text {f }}$, B. Mayes ${ }^{\mathrm{q}}$, J.S. McCarthy ${ }^{\mathrm{m}}$, G. van Middelkoop ${ }^{\mathrm{e}}$, D. Miller ${ }^{\mathrm{p}}$, J. Mitchell ${ }^{\mathrm{m}}$, K. Mori v, J. Moromisato ', G.S. Mutchler ${ }^{b}$, J. Nassalski ${ }^{d}$, L. Naumann ${ }^{x}$, B. Neganov ${ }^{\text {, }}$, T.O. Niinikoski ${ }^{x}$, J.E.J. Oberski e, S. Okumi ${ }^{\vee}$, A. Penzo ${ }^{j}$, G. Perez ${ }^{a}$, F. Perrot-Kunne ${ }^{\text {f }}$, D. Peshekhonov ${ }^{\text {s }}$, R. Piegaia ${ }^{x, 7}$, L. Pinsky ${ }^{q}$, S. Platchkov ${ }^{f}$, M. Plo ${ }^{a}$, D. Pose ${ }^{j}$, H. Postma ${ }^{e}$, T. Pussieux ${ }^{f}$, J. Pyrlik ${ }^{q}$, J.M. Rieubland ${ }^{x}$, A. Rijllart ${ }^{x}$, J.B. Roberts ${ }^{b}$, M. Rodriguez ${ }^{a}$,

E. Rondio ${ }^{d}$, O. Rondon ${ }^{y}$, L. Ropelewski ${ }^{d}$, A. Rosado ${ }^{i}$, I. Sabo ${ }^{z}$, J. Saborido a , G. Salvato ${ }^{j}$,

A. Sandacz ${ }^{\text {d, D. Sanders }}{ }^{4}$, I. Savin ${ }^{\text {s }}$, P. Schiavon ${ }^{\text {j, P. Schüler }}{ }^{k, 8}$, R. Segel ${ }^{\text {p }, ~ R . ~ S e i t z ~ ", ~}$

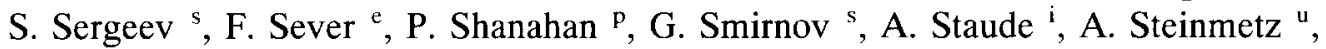

H. Stuhrmann ${ }^{y}$, K.M. Teichert ${ }^{i}$, F. Tessarotto ${ }^{j}$, W. Thiel ${ }^{g, 17}$, Y. Tzamouranis ${ }^{g, 18}{ }^{\text {, M. Velasco }}{ }^{p}$, J. Vogt ${ }^{\mathrm{i}}, \mathrm{R}$. Voss ${ }^{\mathrm{x}}$, R. Weinstein ${ }^{\mathrm{q}}, \mathrm{C}$. Whitten ${ }^{\mathrm{o}}$, R. Windmolders ${ }^{\text {aa }}$, W. Wislicki ${ }^{\mathrm{d}}$, A. Witzmann ${ }^{1}$, A. Yañez ${ }^{\text {a }}$, N.I. Zamiatin ${ }^{\text {s, A.M. Zanetti }}{ }^{\mathrm{j}}$

${ }^{a}$ University of Santiago, Department of Particle Physics, 15706 Santiago de Compostela, Spain ${ }^{9}$

${ }^{b}$ Rice University, Bonner Laboratory, Houston, 77251-1892 TX, USA ${ }^{10}$

'Uppsala University, Department of Radiation Sciences, 75121 Uppsala, Sweden

${ }^{d}$ Warsaw University and Soltan Institute of Nuclear Studies, 00681 Warsaw, Poland ${ }^{n}$

'NIKHEF, Delft University of Technology, FOM and Free University, 1009 AJ Amsterdam, The Netherlands ${ }^{12}$

${ }^{f}$ DAPNLA, CEN Saclay, 91191 Gif-sur-Yvette, France

${ }^{g}$ University of Bielefeld, Physics Department, 33615 Bielefeld 1, Germany ${ }^{13}$

${ }^{h}$ Helsinki University of Technology, Low Temperature Laboratory, Otakaari 3A, 02150 Finland

${ }^{i}$ Unicersity of Munich, Physics Department, 80799 Munich, Germany ${ }^{13}$

${ }^{j}$ INFN Trieste and Unicersity of Trieste, Department of Physics, 34127 Trieste, Italy

${ }^{k}$ Yale University, Department of Physics, New Haven, 06511 CT, USA ${ }^{10}$

${ }^{\prime}$ Unicersity of Freiburg, Physics Department, 79104 Freiburg, Germany ${ }^{13}$,

${ }^{m}$ University of Virginia, Department of Physics, Charlottesville, 22901 VA, USA ${ }^{14}$

${ }^{n}$ Bogaziçi University and Çeknece Nuclear Research Center Istanbul, Turkey

'University of California, Department of Physics, Los Angeles, 90024 CA, USA 10

${ }^{p}$ Northwestern University, Department of Physics, Evanston, 60208 IL, USA ${ }^{10,14}$

* Corresponding author. 
${ }^{q}$ University of Houston, Department of Physics, Houston, 77204-5504 TX, and Institute for Beam Particle Dynamics, Houston, $77204-5506$ TX, USA 10,14

'Northeastern University, Department of Physics, Boston, 02115 MA, USA ${ }^{14}$

${ }^{s}$ JINR, Laboratory of Super High Energy Physics, Dubna, Russian Federation

${ }^{t}$ ETH, 8093 Zürich, Switzerland

"University of Mainz, Institute for Nuclear Physics, 55099 Mainz, Germany 13

"Nagoya University, Department of Physics, Furo-Cho, Chikusa-Ku, 464 Nagoya, Japan ${ }^{15}$

${ }^{w}$ University of California, Institute of Particle Physics, Santa Cruz, 95064 CA, USA

${ }^{x}$ CERN, 1211 Geneva 23, Switzerland

${ }^{y}$ GKSS, 21494 Geestacht, Germany ${ }^{13}$

${ }^{z}$ Tel Aviv University, School of Physics, 69978 Tel Aviv, Israel ${ }^{16}$

${ }^{a}$ University of Mons, Faculty of Science, 7000 Mons, Belgium

Received 4 March 1994

The 10-probe NMR system for measuring the polarization in the deuterated butanol target of the Spin Muon Collaboration is presented. It is calibrated by determining the integrated spectrum in thermal equilibrium at $1 \mathrm{~K}$. Thermal stabilization and control of electromagnetic interference were effective in reducing drift and electronic noise, so that high quality thermal equilibrium signals were obtained. The calibration constant was determined with a reproducibility of $2 \%$. Systematic effects of circuit drift and of the magnetic field shift have been studied. The resulting $4.4 \%$ uncertainty of the calibration dominates the $5 \%$ overall error on the target polarization value which was typically 0.45 .

\section{Introduction}

The NA47 experiment of the Spin Muon Collaboration (SMC) at the CERN SPS aims at measuring the spin dependent structure functions of the proton and the neutron by scattering longitudinally polarized high energy muons from polarized proton and deuteron targets. In the mea-

1 Now at CERN, 1211 Geneva 23, Switzerland.

2 Now at University of Montreal, PQ, H3C 3J7, Montreal, Canada.

${ }^{3}$ Now at Max Planck Institute, Heidelberg, Germany.

${ }^{4}$ Permanent address: Miyazaki University, 88921 Miyazaki-Shi, Japan.

5 Now at PSI, 5232 Villigen-PSI, Switzerland.

6 Permanent address: KEK, 305 Ibaraki-Ken, Japan.

7 Permanent address: University of Buenos Aires, Physics Department, 1428 Buenos Aires, Argentina.

8 Now at SSC Laboratory, Dallas, 75237 TX, USA.

${ }^{9}$ Supported by Comision Interministerial de Ciencia y Tecnologia.

${ }^{10}$ Supported by the Department of Energy.

11 Supported by KBN.

${ }^{12}$ Supported by the National Science Foundation of The Netherlands.

${ }^{13}$ Supported by Bundesministerium für Forschung und Technologie.

${ }^{14}$ Supported by the National Science Foundation.

${ }^{15}$ Supported by Ishida Foundation, Mitsubishi Foundation and Monbusho International Science Research Program.

${ }^{16}$ Supported by the US-Israel Binational Science Foundation and The Israeli Academy of Sciences.

${ }^{17}$ Now at Phillips Kommunications-Industrie AG, Nürnberg, Germany.

${ }^{18}$ Now at University of Virginia, Department of Physics, Charlottesville, VA 22901, USA. surement performed in 1992 , muons of $100 \mathrm{GeV}$ energy were scattered from a longitudinally polarized deuterated butanol target [1]. For this experiment the goal has been to measure the target polarization $P$ with an accuracy $\Delta P / P$ of $5 \%$ or better [2].

The polarized target is the same as the one used in an earlier experiment by the European Muon Collaboration for a measurement with polarized protons $[3,4]$. However, a new NMR system was constructed for the measurement of the deuteron polarization.

In this paper we describe the NMR system and its operating characteristics with emphasis on the error analysis in the measurement of the thermal equilibrium (TE) signal, which provides the calibration for polarization measurement. In addition we present enhanced signals and discuss the precision of the polarization measurement.

Our target is made of $1.8 \mathrm{~mm}$ diameter beads of frozen perdeuterated 1-butanol doped with a deuterated paramagnetic complex EHBA-Cr(V)- $\mathrm{d}_{22}$. The beads are contained in two $40 \mathrm{~cm}$ long cells of $5 \mathrm{~cm}$ diameter. The two cells are separated by a gap of $20 \mathrm{~cm}$ length and the material in them is polarized in opposite directions. Five NMR probes are embedded in the material of each cell.

Dynamic nuclear polarization (DNP) is obtained by microwave irradiation, and it is determined within each probe from the NMR absorption spectrum measured by a series $Q$-meter [5] operating at the deuteron Larmor frequency of $16.350 \mathrm{MHz}$ in a magnetic field of $2.5 \mathrm{~T}$.

The deuteron with spin $I=1$ has three magnetic sublevels with two magnetic dipole transitions, whose spectra are broadened by the quadrupole interaction of the nuclei in the electric field gradient of the molecule. The resulting absorption spectrum of deuterons in glassy hydrocarbons is 
characterized by two overlapping lines with distinct peaks separated by about $125 \mathrm{kHz}$ and a total width close to 300 $\mathrm{kHz}$. In this work these spectra were measured over a frequency scan of $500 \mathrm{kHz}$.

We shall first outline the principles of the NMR polarization measurement in solid targets using the series $Q$-meter circuit, and discuss the techniques of its calibration. In Section 3 we shall describe the NMR probes and electronic circuits, focusing on the key elements and main improvements. The results of the performance tests and calibration runs are given in Section 4, and the polarization measurement is presented in Section 5. In Section 6 we shall conclude and propose future improvements.

\section{Principle of the NMR polarization measurement}

The polarization $P=\left\langle I_{z}\right\rangle / I$ of a spin species $I$ is related to the frequency-dependent, complex susceptibility $\chi(\omega)=\chi^{\prime}(\omega)-i \chi^{\prime \prime}(\omega)$. The real and imaginary parts of this susceptibility describe respectively the dispersion and absorption of the RF field, which excites the magnetization of the spin system. For a system of spins with density $N$ and gyromagnetic ratio $\gamma$, the integral of the absorption part is proportional to the polarization [6]:

$P=\frac{2}{\pi \hbar \gamma^{2} N I} \int_{0}^{\infty} \chi^{\prime \prime}(\omega) \mathrm{d} \omega$.

This general relation is valid even in the limit of high nuclear polarizations, provided that the oscillating RF field is small and has only slow variations of amplitude and frequency.

The integral of the real part $S(\omega)$ of the series $Q$-meter signal is proportional to a good approximation to the integral of $\chi^{\prime \prime}(\omega)$, if the circuit is well designed and tuned. Therefore the use of Eq. (1) requires only the calibration of the apparatus at one known polarization $P_{0}$, which yields

$P=P_{0} \frac{\int_{0}^{\infty} S(\omega) \mathrm{d} \omega}{\int_{0}^{\infty} S_{0}(\omega) \mathrm{d} \omega}=C \int_{0}^{\infty} S(\omega) \mathrm{d} \omega$,

where we have defined the calibration constant

$C=P_{0} / \int_{0}^{\infty} S_{0}(\omega) \mathrm{d} \omega$.

In our case the integrals in Eqs. (1) and (2) get significant contributions only from a narrow range around the Larmor precession frequency, so that the RF susceptibility needs to be measured only in this interval about the resonance.

The methods for determining the polarization $P_{0}$ are based on the measurement of a temperature $T_{0}$ at which the spin system is held during the measurement of the $Q$-meter signal $S_{0}(\omega)$. The Boltzmann distribution of the populations of the magnetic states then gives the value of the polarization $P_{0}$.

In our case $T_{0}$ is the equilibrium temperature of the spins, the lattice and the helium bath around $1 \mathrm{~K}$. At this temperature $P_{0} \ll 1$ and it can be written in the form

$P_{0}=\hbar \omega_{0}(I+1) /\left(3 k_{\mathrm{B}} \mathrm{T}_{0}\right)$,

where $\omega_{0}=\gamma B$ is the Larmor frequency. This TE method requires a short spin-lattice relaxation time which is of the order of $1 \mathrm{~min}$. at $1 \mathrm{~K}$ in our target material. At this temperature the thermal stability and uniformity are excellent when superfluid ${ }^{4} \mathrm{He}$ is used as a heat transfer medium. Also, the international temperature scale ITS 90 is accurate to about $10^{-3}$ in this region and uses the vapor pressure of ${ }^{3} \mathrm{He}$ as a secondary standard, which greatly facilitates thermometry in a high magnetic field.

The work on continuous-wave series $Q$-meter NMR circuit $[5,7-11]$ has focused on its linearity in terms of $\chi$. This becomes important when measuring the polarization of high-density systems of spin-1/2 nuclei with large magnetic moment, such as protons in solid hydrocarbons. The linearity is of minor concern in the case of a broad NMR line, because the RF susceptibility then produces only small changes in the impedance of the tuned circuit of the $Q$-meter. Furthermore, if the gyromagnetic ratio is small, as in the case with deuterons, these changes become even smaller since they scale with the square of the gyromagnetic ratio. In comparison with proton absorption signals the deuteron signal is lower by a factor of about 500 at equal temperature and field, when measured with series $Q$-meters having the same $Q$-factor and RF field strength. The deuteron TE susceptibility causes a relative change of resonant circuit impedance of about $10^{-6}$.

Some authors [12-14] report measurements of the deuteron TE signal by using a very thin bare wire for the NMR coil, in order to increase the signal size. This leads to high RF fields near the wire and the probe is therefore sensitive only to material very close to the wire. The method is not useful for a sizeable target where the average polarization is to be determined over a large volume. Furthermore, strong depolarization of the deuterons near the wire may occur, which leads to significant systematic errors in the polarization measurement.

Averaging is used to improve the signal to noise ratio in all $Q$-meters. We show in Section 4.2 that the electronic noise can be suppressed by a factor more than 100 , so that its contribution to the total error becomes negligible.

However, drift of the $Q$-meter circuit occurs during the averaging time. Indeed, this has been reported [15] to limit the accuracy of the TE calibration signal measurement. We have achieved a substantial reduction in the drift by thermal stabilization (Section 4.3).

The $Q$-meter circuit distorts the NMR absorption spectrum mainly for two reasons: firstly because in a polarized target set-up the coil is connected to the tuning capacitor via a coaxial line, and secondly due to the finite magnitude 


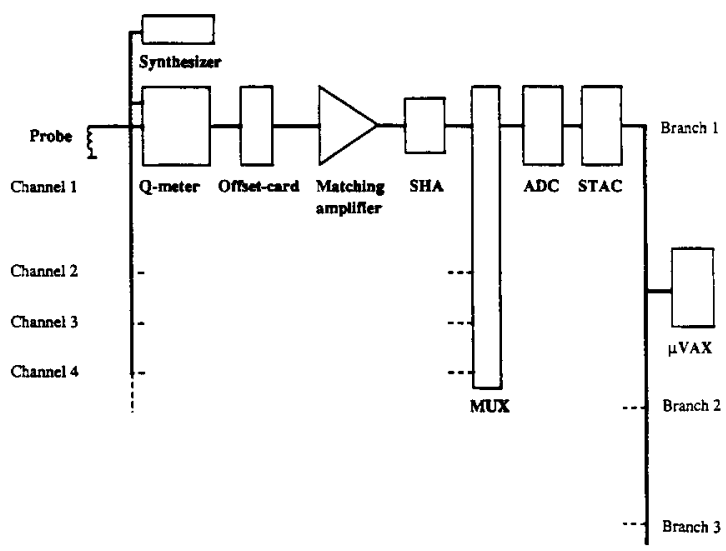

Fig. 1. System block diagram.

of the coupling impedances. Although these distortions are large and visible in the case of the large line widths, they have a linear relationship with the susceptibility, and result only in very small systematic errors if the measurement is calibrated at a known polarization. On the other hand, the linear distortions preclude a straightforward use of the experimental NMR line asymmetry for the accurate determination of the polarization.

Two alternative calibration methods are based either on the measurement of a common equilibrium spin temperature of two different spin species, or on the analysis of the NMR line shape.

The former method relies on the assumption that different nuclear spin species are in good thermal equilibrium after dynamic nuclear polarization. It has been demonstrated that this can be obtained in materials such as propanediol-Cr(V) with high electron spin concentration [16], but tests have shown that in glasses doped with EHBA-Cr(V), such as the material of our target, the spin temperatures of deuterons, protons and ${ }^{13} \mathrm{C}$ can be very different [17].

The latter method has been most often used for determining the deuteron polarization in small targets. After reaching high DNP, $P_{0}$ is obtained by determining the intensity ratio of the two magnetic transitions from the asymmetric shape of the experimental absorption spectrum [18]. This requires that the deuteron spin system is in internal thermal equilibrium and that the material has good

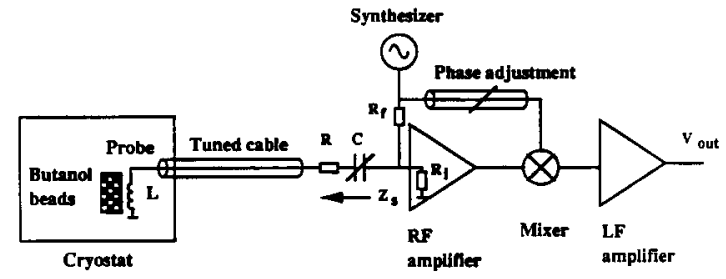

Fig. 2. Schematic diagram of the series $Q$-meter circuit. spin temperature uniformity. Furthermore, good accuracy can be achieved only if $\left|P_{0}\right| \geq 0.3$. In a large target the potential inhomogeneity of the polarization limits the usefulness of this method. Provided that the intensity ratio can be accurately determined from the line shape distorted by the $Q$-meter, it might actually serve better for the estimation of the homogeneity rather than of the polarization itself.

We have chosen the calibration method based on TE signal measurement at $1 \mathrm{~K}$. The line shape was used to put a limit on the inhomogeneity of the DNP.

\section{Electronic circuit description}

\subsection{System overview}

The NMR system consists of $10 Q$-meter channels operating simultaneously. Four channels are multiplexed into one 16 bit $\mathrm{ADC}$, reducing the total number of $\mathrm{ADCs}$ to three.

Each NMR measurement channel, shown schematically in Fig. 1, consists of three basic sections: an RF section, an LF section, and a digital section. The RF section is sub-divided into four parts: the NMR probe coils inside the cryostat, the tuned semi-rigid cables, the $Q$-meter modules, and the digital frequency synthesizer. The frequency of the synthesizer, common to all channels, is scanned in 400 steps through the spectrum of the deuteron spin resonance.

The LF section is sub-divided into two parts: the offset cards and the matching amplifiers. Here the DC level is offset and the remaining signal amplified to a level of several volts, before being sent to the digital section.

The digital section consists of an ADC and a processor. It converts the amplified signal to a 16 bit binary number at each frequency step and accumulates an average over a programmed number of scans.

\subsection{The $Q$-meter module}

The series $Q$-meter circuit of Fig. 2 measures the absorptive part of $\chi(\omega)$. The NMR probes, consisting of three-turn coils of inductance $L=450 \mathrm{nH}$, are embedded in the target material. They are made of thin-wall $\mathrm{Cu}-\mathrm{Ni}$ tubes of $60 \mathrm{~cm}$ length and $2 \mathrm{~mm}$ diameter, surrounded by $3.5 \mathrm{~mm}$ diameter thin-wall Teflon ${ }^{19}$ sleeves. As shown in Fig. 3 the coils are aligned on the axis of the target with a spacing of $4 \mathrm{~cm}$. In order to excite the nuclear spin resonance, they are oriented in such a way as to produce a magnetic field predominantly perpendicular to the main field. Each coil axis is also perpendicular to that of its neighbouring coils to reduce their mutual inductance. The effective probe filling factor is about 0.2 . In addition to

\footnotetext{
${ }^{19}$ Dupont PTFE.
} 


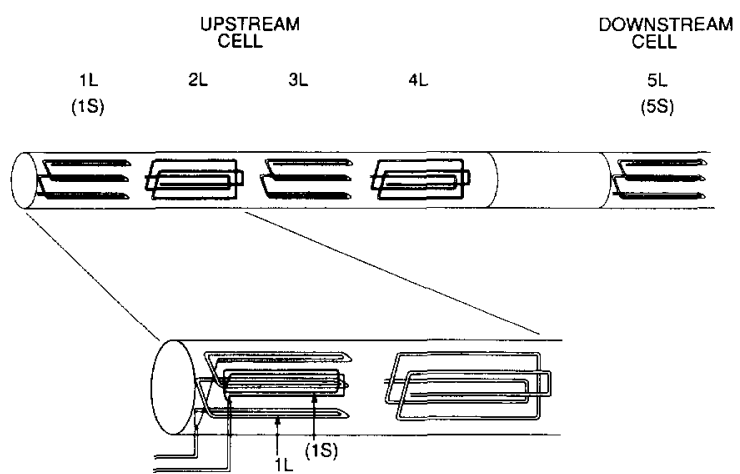

Fig. 3. The large (L) and small (S) coils for deuteron NMR inside the target cell. The bottom part shows an enlargement of the coils $1 \mathrm{~L}$ and $2 \mathrm{~L}$ in the upstream cell.

these large coils there are two smaller ones made of 1.5 $\mathrm{mm}$ diameter $\mathrm{Cu}-\mathrm{Ni}$ tubes again covered with Teflon sleeves of $3.0 \mathrm{~mm}$ diameter. They have two turns, and are $6 \mathrm{~cm}$ long and $1 \mathrm{~cm}$ wide. Each small coil is centered inside a large coil (see Fig. 3) with their axes mutually perpendicular.

Each probe coil in the cryostat is connected to a capacitor $\mathrm{C}$ by a coaxial cable, which is cut to a length corresponding to one half wavelength $(\lambda / 2)$ at the centre frequency of the deuteron NMR. The circuit is tuned to the deuteron NMR frequency by adjusting the tuning capacitor in the $Q$-meter module. The resistor $\mathrm{R}$ provides damping and allows one to achieve a compromise between the requirements set by the dynamic range of the digitizing circuit, the oscillator noise, and the distortion which increases towards the ends of the frequency scan.

The $Q$-meter modules [5] contain all the RF circuitry required for the amplification and homodyne detection of the real part of the signal transferred by the series $Q$-meter circuit. Homodyne detection is obtained in a double balanced mixer with a phased reference signal fed into the local oscillator port. The reference signal is derived by splitting the oscillator signal before the coupling resistor $R_{f}$ feeding the resonant circuit. The reference channel incorporates an adjustable-length delay cable for controlling the phase of the reference signal to the mixer. The module also contains a diode detector (not shown in Fig. 2) which provides an output proportional to the modulus of the $Q$-meter signal; this feature enables the tuning of the circuit without knowledge of the correct phase adjustment for the reference signal.

The outputs of the $Q$-meter module are isolated from the subsequent circuitry by low-noise amplifiers.

\subsection{LF signal processing and data acquisition}

The demodulated $Q$-meter signals are amplified on the offset cards, which have a DAC for each channel for subtracting the DC level of its $Q$-curve so that the full dynamic range of the ADC can be used. This card delivers a balanced signal to the matching amplifier, where the gain can be further adjusted before the sample and hold amplifiers (SHA) to match the maximum input voltage of the ADC modules.

The system has 10 independent channels up to the SHA. A multiplexer between the SHAs and the ADC enables four measurement channels to be accumulated by each of the three processors, thus performing signal averaging in parallel. The extra time required to multiplex is compensated by using a fast $\mathrm{ADC}(10 \mu \mathrm{s}$ conversion time), because frequency stepping times above $100 \mu$ s are necessary to ensure proper settling of the analogue signal circuitry.

The SHAs, the ADC, and the synthesizer interface are on an NMR interface board which connects to a STandAlone CAMAC (STAC) microprocessor [19]. Three of these STAC processors and interface boards take data synchronously by means of a multi-STAC synchronization mechanism [20]. Data averaged over a few thousand scans are sent to a $\mu$ VAX computer via a CAMAC branch. The $\mu V A X$ processes the NMR signals, calculates the target polarization, and stores and displays the signals and the time evolution of the polarization.

\subsection{Temperature stabilization}

The $Q$-meter modules, built of solid copper, were cooled by conduction to a massive mounting plate which is also the bottom of the crate. This plate was cooled by water stabilized at a temperature of $27 \pm 1^{\circ} \mathrm{C}$.

The matching amplifiers, the RF synthesizer and the digital electronics were cooled by forced air flow to $30 \pm$ $1^{\circ} \mathrm{C}$. The air inside the electronic cabinet was recirculated through a heat exchanger cooled by unstabilized water.

The temperature of the coaxial transmission line used in the $Q$-meter circuit was also stabilized. This is important because the dielectric is Teflon, which has an anomalous behaviour between 13 and $19^{\circ} \mathrm{C}$. Manufacturer's data and our measurements [21] indicate that the electrical length of the cable changes by $0.1 \%$ over this temperature range. Furthermore, the propagation constant has a significant hysteresis when the temperature is cycled. In addition, changes of the loss factor with temperature are also minimized.

The temperature of the semi-rigid coaxial lines ${ }^{20}$ between the $Q$-meter modules and the target refrigerator was held at $27^{\circ} \mathrm{C}$ with the same circuit of temperature stabilized water that was used for the cooling of the $Q$-meters and the offset-cards. This is followed by about $1.2 \mathrm{~m}$ of semi-rigid $\mathrm{Cu}-\mathrm{Be}$ coaxial line ${ }^{21}$ along which the temperature changes from ambient to $1 \mathrm{~K}$ during calibration. These

\footnotetext{
${ }^{20}$ Micro-Coax Components, Inc., Type UT-85.

${ }^{21}$ Precision Tube, Inc., Type JT50085.
} 
lines were thermally anchored on five successive heat sinks, cooled by a stabilized flow of helium gas. The last part, made of a miniature semi-rigid line, ${ }^{22}$ connects finally to the probe coil. This part has a length of $0.2-1.2$ $m$ depending on the coil position, and was held at the stabilized calibration temperature of about $1 \mathrm{~K}$ in the mixing chamber of the dilution refrigerator.

\subsection{Electromagnetic interference control}

The $Q$-meter signal is amplified and digitized close to the $Q$-meter output to have a minimum length of LF signal transport and therefore a minimum of electromagnetic interference.

The offset card is placed at the output of the $Q$-meter and inside the crate housing the $Q$-meter. This crate is mounted on top of a shielded rack which contains the RF synthesizer, the NIM bin with the matching amplifiers, and the CAMAC crate housing the microprocessor and digitizing electronics.

The amplified balanced signal is transmitted from the offset card to a matching amplifier by a shielded balanced twisted-pair cable of $3 \mathrm{~m}$ length. The amplifier has a high common mode rejection and its output is connected to the $\mathrm{ADC}$ module by a coaxial cable of $0.6 \mathrm{~m}$ length.

\section{Results of the deuteron TE signal measurements and calibration}

\subsection{Procedure to extract and integrate the experimental signal}

In order to extract the NMR TE signal $S_{0}(\omega)$ from the $Q$-meter output we proceed in three steps as shown in Fig. 4. The NMR signal superimposed on the $Q$-curve (Fig. 4a) is measured at 400 points over the frequency scan from 16.1 to $16.6 \mathrm{MHz}$ and averaged over a large number of scans, usually between 2000 and 10000 . A $Q$-curve alone is then measured with identical number of scans, at a magnetic field offset by $-5 \%$ which shifts the NMR signal outside the frequency scan (Fig. 4b). The first step is to subtract this $Q$-curve from the signal superimposed on the first $Q$-curve. The result shows, in addition to the NMR signal, a residual $Q$-curve (Fig. $4 c$ ). This is removed in a second step by subtracting a parabola fit to 50 points in both wings of the scan. The number of points in the fit is a compromise between statistical accuracy and avoidance of the NMR signal. The processed NMR signal shown in Fig. $4 \mathrm{~d}$ results from this subtraction. The third step is to determine the integrated signal which we obtain by summing all values of $S_{0}\left(\omega_{i}\right)$, and normalizing by the number of points in the scan.

\footnotetext{
${ }^{22}$ Micro-Coax Components, Inc., Type UT-34M.
}

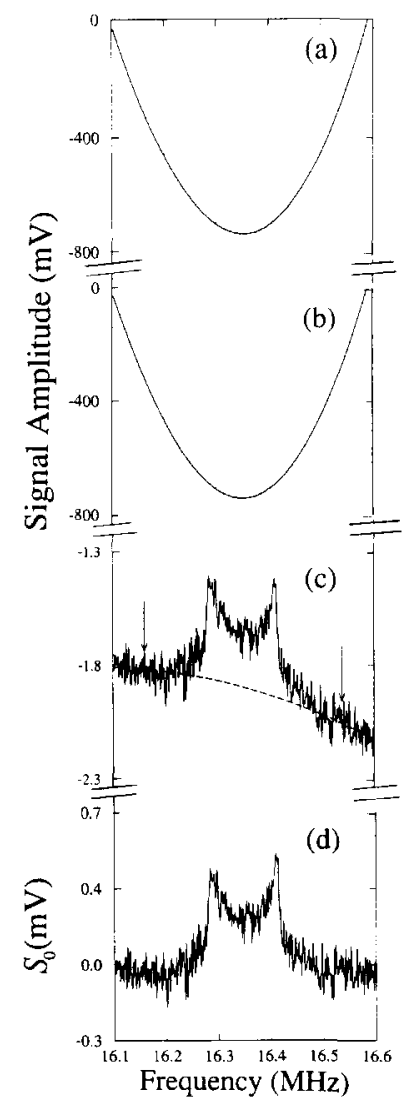

Fig. 4. Method to extract the integrated TE signal from the $Q$-curve, shown with 400 data points averaged over 2000 scans. (a) Plot of the raw measured NMR signal. (b) Plot of the $Q$-curve obtained by shifting the magnetic field. (c) Difference between (a) and (b). (d) Plot of the processed signal where the residual $Q$-curve seen in (c) is corrected by subtracting a parabola fit to the data outside the arrows.

The $Q$-curve has an amplitude about 3000 times larger than the deuteron TE signal under our experimental conditions. The synthesizer has a reproducible frequency dependent amplitude variation about ten times larger than the peak height of the deuteron TE signal; this is subtracted out in the first step of the processing. We are left with noise which is also about ten times higher than the peak height of the signal without signal averaging. The remaining effects on the $Q$-curve are systematic and are due to drift of the $Q$-meter circuitry and to its sensitivity to magnetic field.

\subsection{Electronic signal to noise ratio}

We have estimated the electronic noise by determining the RMS deviation from zero of the processed signal in the fit region of the second processing step described in Section 4.1. Fig. 5 shows the reduction in this RMS noise 


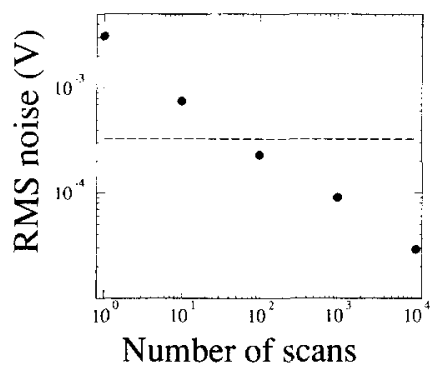

Fig. 5. RMS noise reduction by signal averaging. The noise voltage was obtained from the RMS deviation of the signal voltage from the parabola fit in the wings. The dashed line corresponds to the TE signal mean amplitude at $1 \mathrm{~K}$.

voltage as a function of the number of scans, and demonstrates its statistical nature. However, the 20 minutes required for 10000 scans makes the measurement sensitive to the drift of the circuit. It also takes about 10 minutes to reach a stable field after a current step of $-5 \%$. These and the desire to combine as many measurements as possible in order to stury the systematics, guided us in the choice of 10000 scans for averaging during the TE calibration.

Fig. 6a shows the processed deuteron TE signal at $1 \mathrm{~K}$ averaged over 200 scans. One can barely distinguish its characteristic shape from the noise. Increasing the number

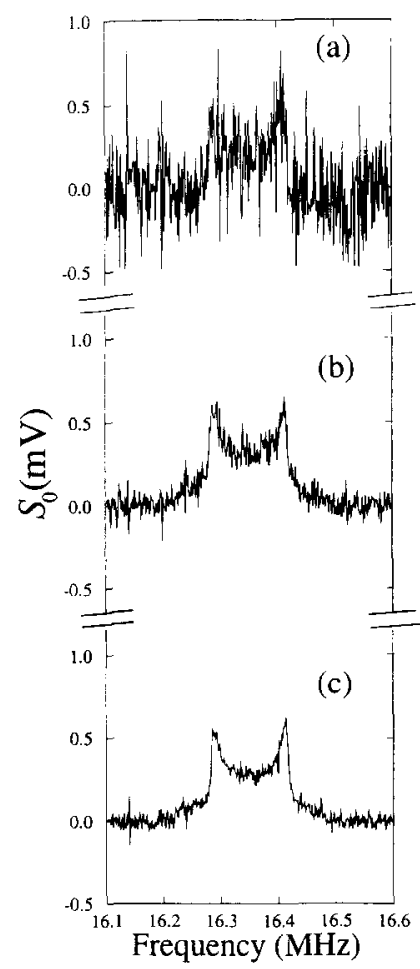

Fig. 6. Processed deuteron TE signals averaged over (a) 200, (b) 2000 and (c) 10000 scans.

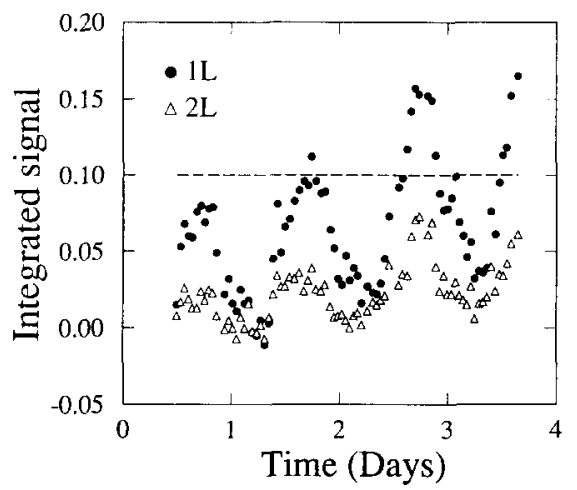

Fig. 7. Variation with time of the integrated residual $Q$-curve. The quantity shown is the integrated processed residual signal, normalised to the integrated TE signal, resulting from the differences between the $Q$-curves and the first $Q$-curve. The periodicity and the long term drift are clearly visible.

of scans to 2000 and 10000 results in the improvement of the signal to noise ratio demonstrated in the Figs. $6 \mathrm{~b}$ and $6 c$, respectively.

\subsection{Stability of the $Q$-curve}

The averaging and $Q$-curve subtraction method requires that there is no substantial drift over the time scale of the signal measurement. In practice drift occurs and leads to a residual $Q$-curve as was discussed in Section 4.1. Subtracting a parabola fit to the wings does not remove higher order terms of this residual $Q$-curve. In the signal integration procedure the contribution of the odd-order terms disappears, but the 4th and higher even-order terms can lead to a finite contribution. To study this, we have recorded over the 4 days period of a TE calibration all the $Q$-curves and subtracted the first from the subsequent ones. The resulting residual $Q$-curves were processed in the same way as the TE signals, by fitting (step 2) and integration (step 3).

These integrated signals are plotted in Fig. 7 as a function of time for coils $1 \mathrm{~L}$ and $2 \mathrm{~L}$ in the upstream cell of the target. A variation, correlated between coils, of the order of the integrated TE signal is observed with a periodicity of one day, superimposed on an increasing trend. The variation for coil $1 \mathrm{~L}$ and coil $8 \mathrm{~L}$ is larger than for all other coils. The periodicity indicates that the variations are caused by temperature fluctuations of the tuned cables and/or the $Q$-meter modules. The differences among the modules, however, suggests that the temperature effect on the $Q$-meter circuits contributes significantly, since the modules for coils $1 \mathrm{~L}$ and $8 \mathrm{~L}$ are located at the two ends of the crate housing the $Q$-meter system.

In TE data processing we subtract successive measurements of signals and $Q$-curves. We have studied the effect of the drift on the processed TE signals using the data of 


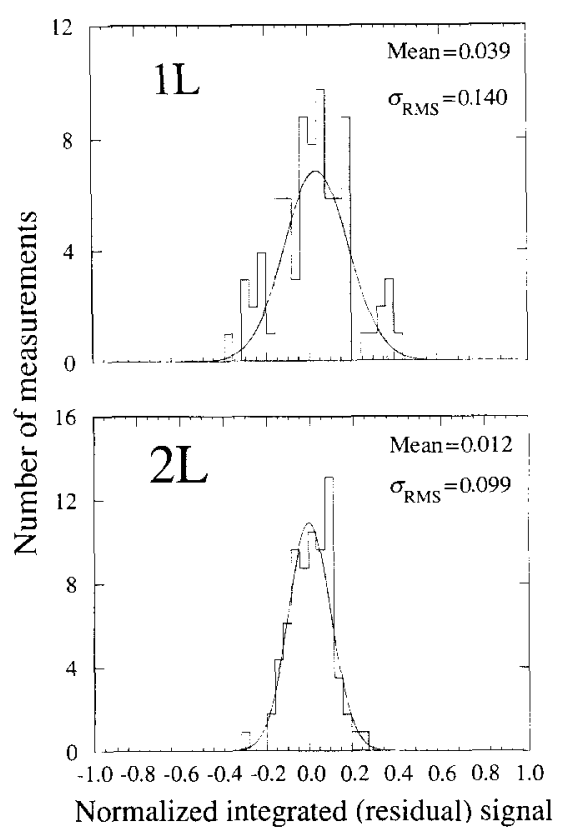

Fig. 8. Distribution of 80 integrated processed residual signals resulting from the differences between successive $Q$-curves, normalized to the TE signal, averaged over 10000 scans. The mean values of these distributions are $3.9 \%$ and $1.2 \%$ respectively, relative to the corresponding integrated TE signals.

Fig. 7. Fig. 8 shows the values of the differences of the successive points in the plot of Fig. 7. The observed distributions have an RMS deviation from the mean of $9.9 \%$ for coil $2 \mathrm{~L}$; similar values are obtained for all other coils except $1 \mathrm{~L}$ and $8 \mathrm{~L}$ for which the RMS is about $14 \%$. This RMS deviation has contributions from the electronic noise and drift at all time scales. The mean value deviates from zero by $\leq 2 \%$ of the integrated TE signal for all coils except $1 \mathrm{~L}$ and $8 \mathrm{~L}$ where the deviations are close to $4 \%$. These deviations are mostly due to the longer term trend visible in the data of Fig. 7. Since the time elapsed between two measurements of $Q$-curves is twice the time between that of a $Q$-curve and a signal, only about half of the effect will remain. We conclude that the drift of the $Q$-meter circuitry introduces a systematic error of about $1.5 \%$ on the volume averaged polarization in each target cell.

\subsection{Magnetic field effect}

Reducing the magnetic field by $5 \%$ to measure the $Q$-curve modifies the impedances of the probe coil and the part of the cable which is exposed to the field. This causes a small shift in the resonant frequency and a change in the $Q$-factor of the tuned circuit and thus contributes to the residual $Q$-curve. In order to study this effect, several $Q$-curves were measured at different fields offset by $-8 \%$ to $+3 \%$ from the nominal value. One signal was measured

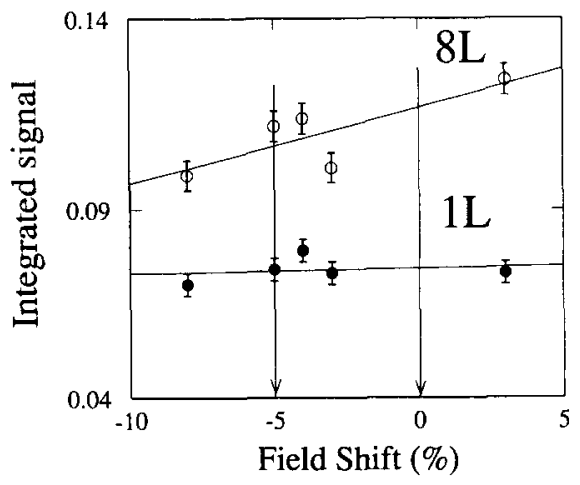

Fig. 9. Dependence of the integrated TE signal on the value of the magnetic field offset when determining the $Q$-curve which is subtracted from the raw NMR signal.

at nominal field, and it was processed by subtracting each one of the $Q$-curves taken at offset fields. The residual $Q$-curves were then removed by fitting (Section 4.1 ). The values of the resulting integrated signals are plotted in Fig. 9 for coils $1 \mathrm{~L}$ and $8 \mathrm{~L}$ as a function of the field shift.

Interpolation of the linear fit gives the magnetic field effect at zero offset, and suggests a correction ranging from $1 \pm 3 \%$ (coil $1 \mathrm{~L}$ ) to $7 \pm 3 \%$ (coil $8 \mathrm{~L}$ ) for the integrated TE signals. The effect increases with coil number and with the cable length exposed to the high field. To distinguish the effect of the field shift from drift due to temperature variation, the data were taken at night during a 2 hour period when the maximum contribution due to drift was about $1 \%$.

When averaged over the upstream and downstream target cells the effect due to the field shift is $4 \%$ which has been taken into account as a systematic error in the TE calibration.

\subsection{Temperature measurement during TE calibration}

The calibration constant $C$ in Eq. (2) requires the accurate knowledge of the TE polarization and therefore that of the temperature, because of their relationship given by Eq. (3). We use the international secondary standard of the ${ }^{3} \mathrm{He}$ vapour pressure scale ITS 90 to define our temperature scale; this scale was transferred to several resistance thermometers in situ in the high field. The resistance thermometers were used for monitoring the stability and uniformity of the temperature during the calibration data taking, and their values were recorded together with that of the vapour pressure thermometer. The relative error on the final calibration constant due to the drift of the temperature was found to be less than $10^{-3}$.

The vapour pressure was measured using a thermally stabilized calibrated capacitive pressure gauge. ${ }^{23}$ The

${ }^{23}$ MKS, Inc. Baratron Type 170 pressure sensor with Type 270B signal conditioner. 
pressure readings were corrected for the thermomolecular effect. Several commercially calibrated resistance thermometers were used for detecting possible thermal gradients in the target held at zero field. None were observed within the $5 \mathrm{mK}$ precision of the thermometer calibrations.

The spin temperature of the target nuclei could be slightly warmer in the beginning of the data taking, because the field was stepped down by $5 \%$ for the measurement of the $Q$-curve between each signal measurement. Estimates based on the measured spin lattice relaxation time give errors well below $1 \mathrm{mK}$.

During the calibration data taking the actual temperature varied in a $50 \mathrm{mK}$ range around the average value of $1.060 \mathrm{~K}$. For the statistical study of the recorded TE signals, the integrated signals were normalized to $1.000 \mathrm{~K}$ spin temperature.

Summing up in squares these errors and the conservative estimates for those due to several other minor sources, we find the resulting precision of about $1 \%$ in the temperature readings entering in the determination of the calibration coefficients.

\subsection{Summary of the errors in the TE calibration}

The distribution of the calibration constants $C_{1}$ and $C_{2}$ for coils $1 \mathrm{~L}$ and $2 \mathrm{~L}$ is shown in Fig. 10 for 80 measurements performed with 10000 scans. We note that the

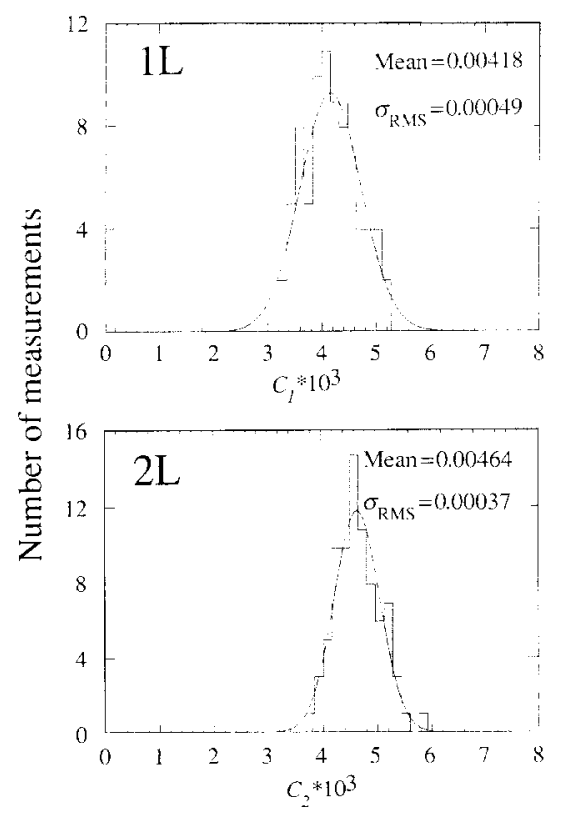

Fig. 10. Distribution of 80 determinations of the calibration constant $C$ for coils $1 \mathrm{~L}$ and $2 \mathrm{~L}$ with 10000 scans.
Table 1

Main sources of errors contributing to the measurement of the calibration constant C, Eqs. (2) and (3), for a single probe

\begin{tabular}{ll}
\hline Source of error & Relative error $(\%)$ \\
\hline Electronic noise and parasitics & 0.1 \\
Temperature measurement & 1.0 \\
Drift of the $Q$-curve & 1.5 \\
Magnetic field effect on the $Q$-curve & 4.0 \\
Total calibration error & 4.4 \\
\hline
\end{tabular}

relative RMS variations from the mean values are different. The larger value for coil $1 \mathrm{~L}$ is a likely consequence of the larger $Q$-curve instability demonstrated in Section 4.3. The calibration constants also appear correlated between coils. Drift of the $Q$-meter, studied and discussed in Section 4.3, explains this correlation. Therefore averaging over the 80 measurements will lead to an accuracy which is inferior to the value of $1 \%$ expected from Fig. 10, if statistical fluctuations were the only cause of the scatter in the values plotted.

After averaging the results for 80 signals, the error in the deuteron TE calibration constants is dominated by systematic uncertainties. The errors, due to the main sources discussed in the Sections 4.2-4.5, are summarized in Table 1 . We estimate the overall relative error to be $4.4 \%$ on the calibration coefficient for each coil, obtained by combining the listed uncertainties quadratically.

In practice we performed several calibrations, two of which covered the polarization measurement during the data taking in 1992. These were made with an interval of 3 months and are denoted by I and II below. As there were no circuit changes between the runs I and II, we can obtain an external consistency check by the comparison of the calibration constants $C_{i}^{\mathrm{I}}$ and $C_{i}^{\mathrm{II}}$, listed in Table 2 . The

Table 2

Calibration constants obtained in runs I and II performed with an interval of 3 months

\begin{tabular}{llccl}
\hline Coil number $i$ & Coil type & $C_{i}^{\mathrm{I}} \times 10^{3}$ & $C_{i}^{\mathrm{II}} \times 10^{3}$ & $C_{i}^{\mathrm{I}} / C_{i}^{\mathrm{II}}$ \\
\hline 1 & $\mathrm{~L}$ & 4.17 & 4.18 & 0.998 \\
2 & $\mathrm{~L}$ & 4.74 & 4.64 & 1.020 \\
3 & $\mathrm{~L}$ & 4.39 & 4.16 & 1.056 \\
4 & $\mathrm{~L}$ & 4.73 & 4.67 & 1.013 \\
5 & $\mathrm{~L}$ & 4.71 & 4.69 & 1.004 \\
6 & $\mathrm{~L}$ & 4.32 & 4.45 & 0.970 \\
7 & $\mathrm{~L}$ & 4.41 & 4.44 & 0.994 \\
8 & $\mathrm{~L}$ & 3.69 & 3.76 & 0.981 \\
1 & $\mathrm{~S}$ & 9.46 & 9.78 & 0.968 \\
5 & $\mathrm{~S}$ & 10.29 & 10.15 & 1.014 \\
Average value & & & & \\
$\quad$ for coils 1L-8L & & & & 1.005 \\
\hline
\end{tabular}


average of the ratios $C_{i}^{\mathrm{I}} / C_{i}^{\mathrm{II}}$ for coils $1 \mathrm{~L}$ to $8 \mathrm{~L}$ is 1.005 with a RMS deviation of $2.5 \%$. The differences for individual coils ate thus compatible with the drift of their $Q$-curves, and we conclude that ageing and other causes of long term drift produce changes which are small in comparison with the observed short term drift.

\section{Determination of deuteron polarization}

\subsection{Treatment of the enhanced signals}

When measuring polarization, the adjustable parameters of the NMR system are identical to those used for the TE signal measurement. They include the $Q$-meter tuning capacitor, the gain factor, the RF frequency and scan width. This procedure minimizes possible systematic errors in the ratio between the two integrated signals in Eq. (2). However, because of the larger signal size, the averaging for noise reduction needs only 200 scans for both $Q$-curve and signal.

The processing of the enhanced NMR signal is identical to that of the TE signal. The polarization is then

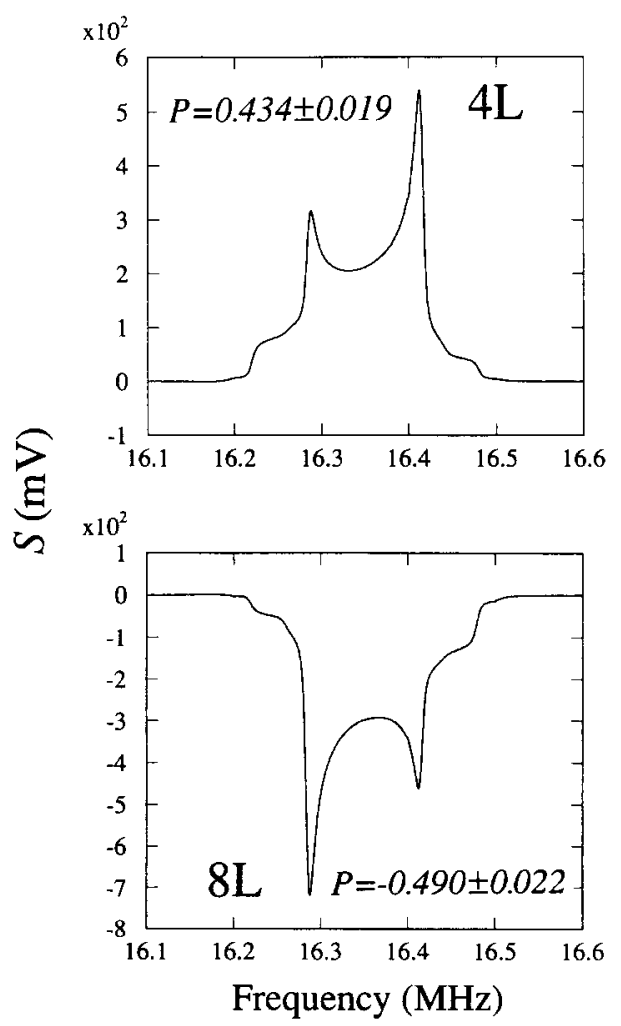

Fig. 11. Typical deuteron signals for enhanced positive and negative polarizations with 200 scans.

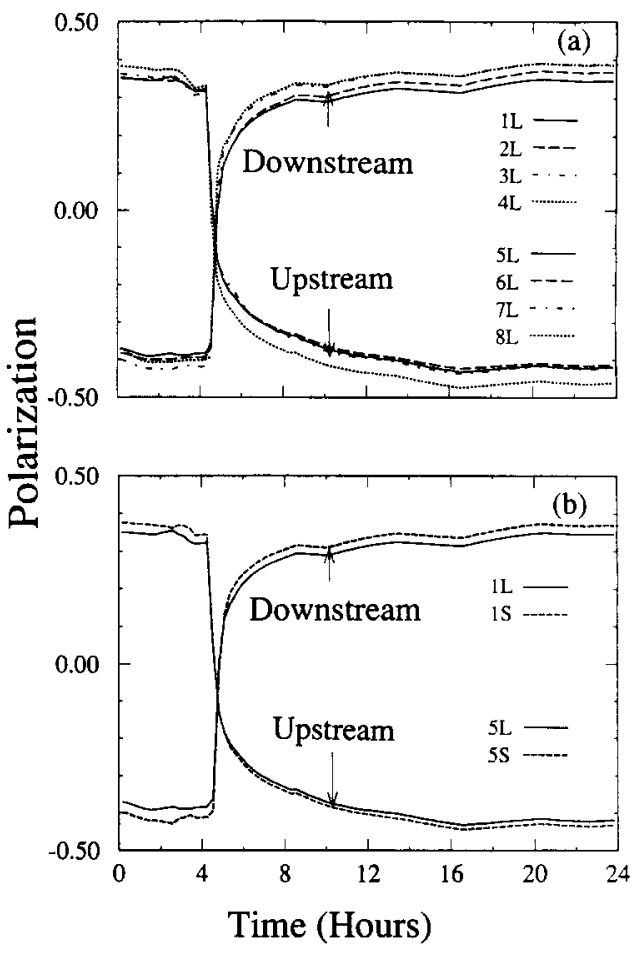

Fig. 12. Time evolution of polarization over a 24 hour period (a) for the 8 large probes and (b) for the concentric large and small probes in both the upstream and the downstream target cells.

deduced from the value of the integrated signal using the calibration constants $C_{i}$. The enhanced signals of Fig. 11 correspond to polarization values of $P=0.434 \pm 0.019$ and $P=-0.490 \pm 0.022$. These high values were routinely obtained during the SMC experiment after it was discovered that modulation of the polarizing microwaves almost doubled the polarization in our large target. The quoted errors correspond to the $4.4 \%$ uncertainty in the calibration constants.

\subsection{Averaging of the polarization and resulting error}

The target polarization was continuously monitored; Fig. 12 shows a typical evolution of polarizations over a period of 24 hours including a reversal by DNP. We note small differences in the time constants of polarization growth for the different probes. As seen in Fig. 12a, significant differences in polarization up to $15 \%$ remain between the probes when we reach high polarizations. This feature is present in both cells and for both signs of polarization.

A limit for the inhomogeneity of polarization can be obtained from the deuteron line shape as discussed in Section 2. Provided that the polarization is uniform and 
that the spin system is in internal thermal equilibrium in the volume sampled by the coil, $P$ and the transition intensity ratio $\mathrm{r}$ are related by [18]

$P=\left(r^{2}-1\right) /\left(r^{2}+r+1\right)$.

On the other hand the experimental intensity ratio $r^{*}$ can be obtained from the NMR spectrum. We determined $r^{*}$ assuming that the distortions of the spectrum are linear in terms of the frequency. The values of $r^{*}$ in dynamic equilibrium were always larger (smaller) than $r$ for positive (negative) polarization; the difference ranges from $5 \%$ to $10 \%$ and is marginally significant. From the precision of the determination of $P$ and $r^{*}$ we can put a limit $|\Delta P|$ $\leq 0.15$ on the spatial variation of the polarization in the volume sampled by each probe.

The analysis of the deep inelastic data requires the averaged polarization for each target cell over periods of few hours. Only data collected with $|P| \geq 0.25$ were used. The time averaging of polarization was performed using the NMR data recorded every two minutes. A proper spatial sampling of the polarization should weight the target in a particular region in the same way as the muon beam does. The sampling by the beam differs from that by the NMR probe, mainly due to the steeper radial variation of the muon beam density. The average polarization value is taken as the average of the four polarization values given by the large NMR probes of each target cell. Fig. $12 \mathrm{~b}$ shows that the polarizations measured by the small coils $1 \mathrm{~S}$ and $5 \mathrm{~S}$ are $\approx 6 \%$ larger than the polarizations measured by the surrounding concentric large coils $1 \mathrm{~L}$ and $5 \mathrm{~L}$. The effect of a possible radial variation of polarization was estimated using a simulation and making extreme assumptions on the radial polarization gradient compatible with the differences between the concentric coils. The simulation leads to an additional uncertainty of $2 \%$. No error was assigned to the sampling variation of the NMR probes in the axial direction. The overall relative error of the average polarization for each target cell is therefore $5 \%$.

\section{Summary}

In conclusion our NMR system based on a well designed series $Q$-meter and used with careful attention to systematic errors has achieved a relative overall accuracy of $5 \%$ in the measurement of the deuteron polarization for the large deuterated butanol target of the SMC experiment at CERN. Further improvement in the accuracy can be achieved by additional study of the magnetic field effect on the $Q$-curves for the TE calibration and also of the NMR line shape which provides information on the spatial inhomogeneity of the target polarization. Average deuteron polarization values of 0.45 were routinely obtained.

\section{Acknowledgements}

We are grateful to $\mathrm{R}$. Broughton, R. Campergue, G. Court, G. Durand and G. Gallay for technical support during the various phases of the fabrication and tests of the apparatus.

\section{References}

[1] SMC, B. Adeva et al., Phys. Lett. B 302 (1993) 533.

[2] SMC, G. Baum et al., Proposal CERN/SPSCS 88-47 (SPSC/P242) (1988).

[3] EMC, J. Ashman et al., Nucl. Phys. B 328 (1989) 1.

[4] S.C. Brown et al., Proc. 4th Int. Workshop on Polarized Target Materials and Techniques, ed. W. Meyer, University of Bonn (1984) p. 102.

[5] G. Court, D.W. Gifford, P. Harrison, W.G. Heyes and M.A. Houlden, Nucl. Instr. and Meth. A 324 (1993) 433.

[6] M. Goldman, J. Magn. Res. 17 (1975) 393.

[7] C. Ryter, Nucl. Instr. and Meth. 49 (1967) 267.

[8] V. Petricek and M. Odehnal, Nucl. Instr. and Meth. 52 (1967) 197

[9] V. Petricek, Nucl. Instr. and Meth. 58 (1968) 117.

[10] F. Udo, Proc. 2nd Int. Conf. on Polarized Targets, ed. G. Shapiro, LBL, University of California, Berkeley (1971) p. 397.

[11] T.O. Niinikoski, Proc. 2nd Workshop on Polarised Target Materials, eds. G.R. Court, S.F.J. Cox, D.A. Cragg and T.O. Niinikoski, Report RL-80-080, SRC, Rutherford Laboratory (1980) p. 80.

[12] W. Meyer et al., Nucl. Instr. and Meth. 204 (1982) 59; 227 (1984) 35; A 244 (1986) 574

[13] G. Wait et al., Nucl. Instr. and Meth. A 274 (1989) 515.

[14] E. Gülmez et al., Phys. Rev. C 45 (1991) 22.

[15] D. Gifford, Proc. 2nd Workshop on Polarised Target Materials, eds. G.R. Court, S.F.J. Cox, D.A. Cragg and T.O. Niinikoski, Report RL-80-080, SRC, Rutherford Laboratory (1980) p. 85.

[16] W. de Boer, M. Borghini, K. Morimoto, T.O. Niinikoski and F. Udo, J. Low Temp. Phys. 15 (1974) 249.

[17] T.O. Niinikoski, Proc. 2nd Workshop on Polarised Target Materials, eds. G.R. Court, S.F.J. Cox, D.A. Cragg and T.O. Niinikoski, Report RL-80-080, SRC, Rutherford Laboratory (1980) p. 60.

[18] K. Scheffler, Proc. 2nd Int. Conf. on Polarized Targets, ed. G. Shapiro, LBL, University of California, Berkeley (1971) p. 271.

[19] T.O. Niinikoski and A. Rijllart, Nucl. Instr. and Meth. 199 (1982) 485.

[20] A. Rijllart, T.O. Niinikoski, N. Hayashi and R. Ent, Proc. Solid Polarized Target Workshop of the 9th Int. Symp. on High Energy Spin Physics, Vol. 2, eds. W. Meyer, E. Steffens and W. Thiel, Bonn (1990) p. 272.

[21] S. Dhawan, IEEE Trans. Nucl. Sci. NS-39 (1992) 1331. 